\title{
Erratum
}

\section{Optical Properties of Dense Exciton-Biexciton Systems}

R. März, S. Schmitt-Rink, and H. Haug

Z. Phys. B - Condensed Matter 40, 9-14 (1980)

Equations (2) and (3) should be:

$\varepsilon(\mathbf{k}, \omega)=\varepsilon_{\infty}-\left|g_{\mathbf{k}}\right|^{2} D_{x}^{R}(\mathbf{k}, \omega)$,

where $D_{x}^{R}(\mathbf{k}, \omega)$ is the retarded exciton Green's function

$D_{x}^{R}(\mathbf{k}, \omega)=\frac{1}{\omega-\omega_{x}+i \varepsilon}-\frac{1}{\omega+\omega_{x}+i \varepsilon}$.

The time-ordered Green's function, which is given in (3) is used to calculate the self-energy due to biexciton formation.

Equation (8) should be:

$$
\begin{aligned}
& \varepsilon(\omega)=\varepsilon_{\infty} \\
& +\frac{\left(\varepsilon_{0}-\varepsilon_{\infty}\right) \omega_{x}^{2}\left(z_{M}-2 \omega\right)}{\left(z_{M}-2 \omega\right)\left(\omega_{x}^{2}-\omega^{2}\right)-\Omega^{2} \omega_{x}\left(z_{M}-\omega\right) / z_{M}} .
\end{aligned}
$$

All numerical results remain unchanged.

R. März

S. Schmitt-Rink

H. Haug

Institut für Theoretische Physik

Universität Frankfurt/Main

Robert-Mayer-Strasse 8

D-6000 Frankfurt/Main 1

Federal Republic of Germany 